American J. of Engineering and Applied Sciences 4 (2): 265-269, 2011

ISSN 1941-7020

(C) 2011 Science Publications

\title{
Influence of Organic Wax on Bitumen Characteristics
}

\author{
Arafat Suleiman Yero and Mohd Rosli Hainin \\ Construction Research Alliance \\ Department Geotechnics and Transportation, Faculty of Civil Engineering, \\ Universiti Teknologi, Skudai, Johor, Malaysia
}

\begin{abstract}
Statement problem: The study investigated effect of organic wax Sasobit wax (S) on the characteristics of bitumen 80/100-penetration grade. The consistency of bitumen is measure of its susceptibility to temperature change and resistance to flow which affects ability and resistance to deformation of the mixture. Approach: This study reviewed the bitumen modification process in relation to Warm Mix Asphalt (WMA) technology, using $\mathrm{S}$ as a modifier. The study investigated the penetration, softening point and viscosity measurements of modified bitumen 80/100-penetration grade (binder), using the Brookfield viscometer. The binders mixed with various percentage of the wax S 1-5\% were investigated. Results: Results from the study showed an increase in softening point, decrease in penetration with an increase in S. The viscosity of binder also decreases at higher temperatures while at midrange temperatures the viscosity increases with an increase in additive. Conclusion: This study has provided a valuable data on the effect of additive $S$ on increasing the kinematic viscosity of binder at low temperature and decreasing the dynamic viscosity at high temperature, been attributed to the presence of $\mathrm{S}$ wax with high hydrocarbons molecular content in the binder. Also increasing the additive decreases penetration and increases softening points, The study recommends the use of $2 \% \mathrm{~S}$ for modification of PEN 80/100.
\end{abstract}

Key wards: Sasobit wax, softening point, Warm Mix Asphalt (WMA)

\section{INTRODUCTION}

Bitumen is a thermo-visco-elastic material where temperature and rate of load application have a great influence on their behavior. They are classified as rheological materials since their viscosity stress and strain response is both time and temperature dependent. The binder consistency and hence ability to sustain and hold its fundamental cementing mechanism changes depending on its viscosity. In the study bitumen with penetration grade 80/100 were modified with Sasobit wax (S), which is obtained from coal gasification using the Fischer-Tropsch process. According to Kim, (2010) Sasobit has a long-chain hydrocarbon content range $\mathrm{CH}_{40}-\mathrm{CH}_{115}$.

The viscosity of bitumen is its internal resistance to flow or measure of it resistance to deformation by either shear stress or tensile stress and a significant parameter in determining the bitumen rheology and engineering properties of asphalt concrete; it influences the ability and resistance to mix.

The empirical parameters such as penetration, softening points and viscosity where often use to categories the bitumens. The viscosity of bitumen shows a complex response under different conditions and it influences the mixing, laying and compaction of asphalt mixtures as well as the pavement performance. Inview of these reasons viscosity, penetration and softening point measurements have been used to classify bitumen, but due to the visco-elastic nature of bitumens the results lack uniformity as the bitumen source and molecular content might differ (Xiaohu and Isacsson, 2006).

Most of the roads in Malaysia are constructed as asphalt concrete pavements, which mainly consist of bitumen and aggregate. The bitumen though in smaller quantity as compared to the aggregate serves as a binder to the pavement materials. The recent increase in traffic volume, resulting to intense loading of the pavements across the country with severe consequences leading to permanent deformation of the pavement is of great concern. This has prompted a strong interest in developing alternative means of enhancing the performance of the bitumen used in the production of asphalt concrete, these include modification of the binder with either organic additives like $\mathrm{S}$ or polymers.

Bitumen is a rheological, thermoplastic and viscoelastic material. Its deformation haracteristics vary not only with load, but also with time rate of load application and temperature. It is neither lastic nor viscous in behavior. At low temperature it exhibits elastic behavior while at high temperature it exhibits viscous behavior. The neat binders lack the proper viscous-elastic balance that

Corresponding Author: Arafat Suleiman Yero, Department of Highway/Transportation, Faculty Civil, University Technology Malaysia, Skudai, Johor Malaysia Tel: +60177218143 
usually occurs due to an effective elastic structure created by molecular association. It is hypothesized that proper visco-elastic balance can be achieved by the modification of bitumen to enhance its performance in pavements (Marandi and Safapour, 2009).

The performance of asphalt pavement is mainly governed by the properties of the binder. Bitumen exhibit's a visco elastic behavior hence in pavement when exposed to high temperature permanent deformation (Rutting) takes place along the wheel path of the pavement. On the other hand, bitumen in pavement at low temperature exhibits brittleness and pavement cracking occurs.

Most studies on bitumen modification is related to the use of polymer not much has been done on the use of waxes such as $\mathrm{S}$ in bitumen. A study by Sasol International, 2004 indicated a significant drop in the viscosity of the binder when modified with $\mathrm{S}$ at high temperature and hardening at low temperatures. Polymers and additives are oftenly used to enhance the performance of bitumen and experimentation with the use of $\mathrm{S}$ in asphalt concrete started in early 1990s and this technology called Warm Mix Asphalt (WMA) has been claiming to improve the engineering properties of bitumen, such that in cool paving WAMfoam are used in countries like Norway Gandhi and Amirkhanian (2008). Early research and marketing efforts have mainly focused on the environmental benefits and the reduced energy consumption of the technology and not as much on the viscosity and consistency of the modified binder at high and low temperature. This study investigated the viscosity at production and temperature of neat and modified bitumens.

In this study the commercial additive $\mathrm{S}$ was used to lower viscosity of the binder at high temperature, making it less susceptible to high temperature damage and flow improver at low temperature.

The additive $\mathrm{S}$ is introduced to Warm Mix Asphalt (WMA) by blending with the binder at the terminal or contractor's tank. The recommended addition rate is $0.8-3 \%$ by mass of the binder according Sasol report 2004. At high temperatures $S$ lowers the viscosity of the binder such that temperatures decrease by $45^{\circ} \mathrm{C}$ Gandhi and Amirkhanian (2008). The additive has a congealing temperature of about $100^{\circ} \mathrm{C}$ and is completely soluble in binders at temperatures lower than $110^{\circ} \mathrm{C}$, at temperatures below its melting point, it forms a crystalline structure in the binder that is reported to provide added stability as the bitumen stiffens at low temperature.

Previous research: A study by Maccorone, (1994) examined developments in cold mixed asphalt based on the use of foamed bitumen and very high binder content emulsions. He wrote that around the World the use of cold mixes for use on roads are gaining greater acceptance. Such systems are energy efficient and environmentally friendly. Cold mixes do not emit hydrocarbons and use less fuel in manufacturing.

Modification of the binder has relatively resulted in the development of Warm Asphalt Mix process (WAM) technology in Europe. Various studies were undertaken describing an innovative warm mixture process that was tested in the laboratory and evaluated in large-scale field trials (in Norway, the UK and the Netherlands) with particular reference to the production and laying of dense graded wearing courses. Their study resulted in the development of WAM Foam, Warm Asphalt Mix with foamed bitumen (Kim, 2010).

Also previous studies on zeolite additives when added in WMA mix creates a foaming effect that easies the mixing process. Warm mixes have received much attention in Europe and Australia since around the year 2000 (Brian and Graham, 2005).

A study by Edwards, et al., 2006, investigated the effect of commercial wax on the rheological proerties of bitumen 160/220-grade penetration at low temperature. Their study dealt with the low temperature effects, which can influence the thermal cracking resistance of the asphalt concrete. The study indicated the magnitude and type of effect on the bitumen rheology depends on the bitumen type as well as the type and quantity of the additive used.

The effect of commercial wax on the rheological properties of bitumen 60/220-grade penetration at high and medium temperature were investigated by Edwards et al., 2006. Their study dealt with the high and medium temperature effects, which can influence the rutting resistance of the asphalt concrete.

The study indicated the magnitude and type of effect on the bitumen rheology depends on the bitumen type as well as the type and quantity of the additive used, as adding polyethylene wax to the binder has showed considerable changes on the rheological properties of the binder at medium and high temperatures. 
A study to investigate the potentials of warm mix asphalt additives was conducted by Kanitpong et al. 2008. They examine the effect of Sasobit (long-chain aliphatic hydrocarbon) when used as an asphalt modifier, on the rheological properties, viscosity, rutting resistance and fatigue resistance. They also investigated the compatibility of the binder with $S$ at low temperature and also the effect of $\mathrm{S}$ on the performance of the binder mixtures produced at low temperatures.

Meor et al. 2010 investigated the potential of sasobit to reduce heat energy and $\mathrm{Co} 2$ emission in the asphalt industry. They quantify the reduction in fuel consumption due to the reduction in mixing and compaction temperatures in the production of asphalt concrete using Sasobit modified binder.

However, there are relatively no extensive investigation on the characteristics of modified bitumen consistency and their viscosity property, which is oftenly use in asphalt concrete mixtures in Malaysia. Hence this study presents the effect of $\mathrm{S}$ when added to the binder relative to the viscosity, softening point and penetration.

\section{MATERIALS AND METHODS}

For this study thirty samples of bitumen PEN 80/100 grade obtain from the UTM highway laboratory (unknown source) and pellets of Sasobit wax were used for the study. The binder tests and classification is presented in Table 1; Each sample contains $300 \mathrm{~g}$ of bitumen PEN80/100 was obtained from the container after heating at $150^{\circ} \mathrm{C}$ for $3 \mathrm{~h}$, the additive was added at test quantities and a mechanical stirrer at $800 \mathrm{rmp}$ for $15 \mathrm{~min}$ was used to mix it up. Neat and modified bitumen totally 30 samples, with various percentage of S (1-5\%) were investigated in the study.

Penetration and softening point: The penetration grade of the neat bitumen with and without $\mathrm{S}$ was investigated using the penetrometer in accordance with ASTM D5-86 (2006) specification, while the softening point of the various test samples was determined using the ring and ball test in accordance to ASTM D36-95 specifications.

Table 1: Bitumen test and classification

\begin{tabular}{ll}
\hline Test & ASTM bitumen \\
\hline Penetration $(\mathrm{dmm})$ & D5-86 \\
& $80 / 100$ \\
Softening point $(\mathrm{C})$ & D36-95 \\
& 46 \\
\hline
\end{tabular}

Viscosity: The viscocity of the binder was measured using the Brookfield viscometer; the equipment was used to measure the viscosity characteristics of the neat and modified binder PEN 80/100 (with and without S).

This study determined influence of the additive on the viscosity of the binder at midrange and high temperatures, which. The viscosity of each bitumen sample with and without $\mathrm{S}$ was measured at various test temperatures and at a shear rate of 6.8/s. This shear rate was selected because it conforms to the rotational speed of 20rpm with the Brookfield Spindle 27 recommended for Superpave (SHRP). The viscosity of the bitumen with and without Sasobit were measured at $135^{\circ} \mathrm{C}, 75^{\circ} \mathrm{C}$ instead of $60^{\circ} \mathrm{C}$ as required by SHRP, othis was due to hardening of the modified binder.

\section{RESULTS}

The study investigated the viscosity of bitumen $80 / 100$ with various $\%$ of additive by the means of the Brookfield viscometer. The primary objective of the study was to determine the penetration, softening point and viscosity shear stress of the modified bitumen. The binder consistency test is presented in Table 2 below;

There was a decrease in viscosity of the binder at high temperature as the Sasobit was increased and this could be attributed to the presence of the additive. It can also be seen from the Table 3 and 4 that the addition of the additive reduced the viscosities of the various binder mixes at $135^{\circ} \mathrm{C}$ and $150^{\circ} \mathrm{C}$ respectively.

Table 2: Bitumen consistency test

\begin{tabular}{llllll}
\multicolumn{3}{l}{ Bitumen Bitumen } & Bitumen & \multicolumn{4}{l}{ Bitumen Bitumen } \\
Neat & +1 & +2 & +3 & +4 & +5 \\
Bitumen & $\%$ saso & $\%$ saso & $\%$ saso & $\%$ saso & $\%$ \\
$80 / 100$ & $60 / 70$ & $40 / 50$ & $30 / 40$ & $20 / 30$ & $10 / 20$ \\
& & & & & \\
46 & 47 & 52 & 65 & 71 & 82 \\
\hline
\end{tabular}

Table 3: Bitumen $80 / 100$ Viscosity at $135^{\circ} \mathrm{C}$

\begin{tabular}{lllllll}
\hline & $\mathrm{B}+$ & $\mathrm{B}+$ & $\mathrm{B}+$ & $\mathrm{B}+$ & $\mathrm{B}+$ & $\mathrm{B}+$ \\
$\mathrm{Des}$ & $0 \% \mathrm{~S}$ & $1 \% \mathrm{~S}$ & $2 \% \mathrm{~S}$ & $3 \% \mathrm{~S}$ & $4 \% \mathrm{~S}$ & $5 \% \mathrm{~S}$ \\
\hline $\mathrm{CP}$ & 410.0 & 400.0 & 300.0 & 200.0 & 200.0 & 100.0 \\
$\mathrm{SS}$ & 27.9 & 27.2 & 20.4 & 13.6 & 13.6 & 6.8 \\
$\mathrm{SR}$ & 6.8 & 6.8 & 6.8 & 6.8 & 6.8 & 6.8 \\
$\mathrm{~T} \%$ & 0.41 & 0.4 & 0.3 & 0.2 & 0.2 & 0.1 \\
\hline
\end{tabular}


Am. J. Engg. \& Applied Sci., 4 (2): 265-269, 2011

Viscosity@ $150^{\circ} \mathrm{C}$

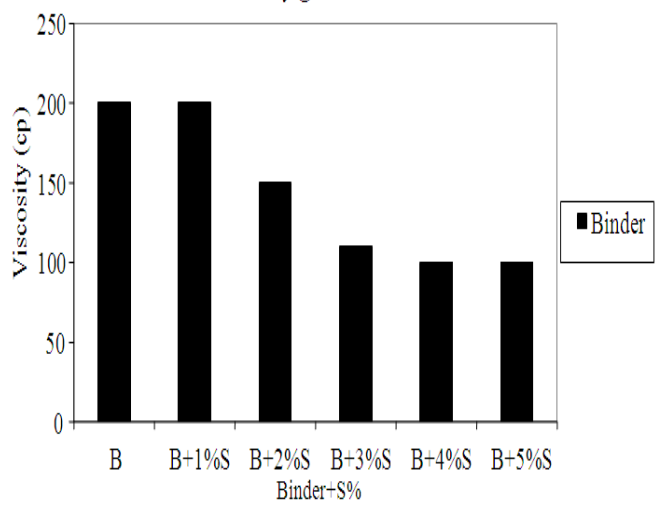

Fig. 1: Viscosity at high temperature

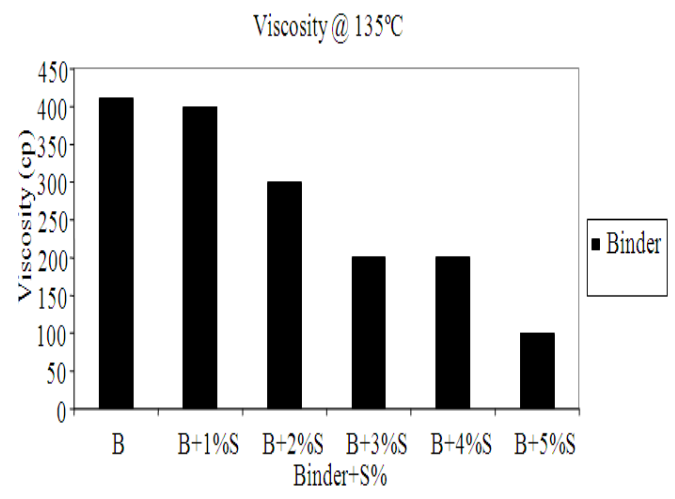

Fig. 2: Binder viscosity

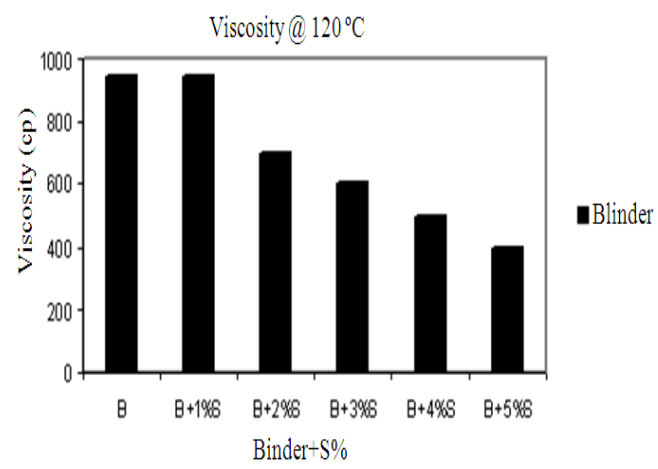

Fig. 3: Viscosity at $120^{\circ} \mathrm{C}$

Table 4: Bitumen $80 / 100$ Viscosity at $150^{\circ} \mathrm{C}$

\begin{tabular}{lllllll}
\hline & $\mathrm{B}+$ & $\mathrm{B}+$ & $\mathrm{B}+$ & $\mathrm{B}+$ & $\mathrm{B}+$ & $\mathrm{B}+$ \\
Des. & $0 \% \mathrm{~S}$ & $1 \% \mathrm{~S}$ & $2 \% \mathrm{~S}$ & $3 \% \mathrm{~S}$ & $4 \% \mathrm{~S}$ & $5 \% \mathrm{~S}$ \\
\hline $\mathrm{CP}$ & 300.0 & 300.0 & 200.0 & 200.0 & 100.0 & 100.0 \\
$\mathrm{SS}$ & 20.4 & 20.4 & 13.6 & 13.6 & 6.8 & 6.8 \\
$\mathrm{SR}$ & 6.8 & 6.8 & 6.8 & 6.8 & 6.8 & 6.8 \\
$\mathrm{~T} \%$ & 0.3 & 0.3 & 0.2 & 0.2 & 0.1 & 0.1 \\
\hline
\end{tabular}

Table 5: Bitumen $60 / 70$ Viscosity at $120^{\circ} \mathrm{C}$

\begin{tabular}{lllllll}
\hline & $\mathrm{B}+$ & $\mathrm{B}+$ & $\mathrm{B}+$ & $\mathrm{B}+$ & $\mathrm{B}+$ & $\mathrm{B}+$ \\
Des & $0 \% \mathrm{~S}$ & $1 \% \mathrm{~S}$ & $2 \% \mathrm{~S}$ & $3 \% \mathrm{~S}$ & $4 \% \mathrm{~S}$ & $5 \% \mathrm{~S}$ \\
\hline $\mathrm{CP}$ & 950.0 & 950.0 & 700.0 & 600.0 & 500.0 & 400.0 \\
$\mathrm{SS}$ & 64.62 & 64.62 & 47.6 & 40.8 & 34.0 & 27.2 \\
$\mathrm{SR}$ & 6.80 & 6.8 & 6.8 & 6.8 & 6.8 & 6.8 \\
$\mathrm{~T} \%$ & 0.95 & 0.95 & 0.7 & 0.6 & 0.5 & 0.4 \\
\hline
\end{tabular}

Table 6: Bitumen $80 / 100$ Viscosity at $100^{\circ} \mathrm{C}$

\begin{tabular}{lllllll}
\hline & $\mathrm{B}+$ & $\mathrm{B}+$ & $\mathrm{B}+$ & $\mathrm{B}+$ & $\mathrm{B}+$ & $\mathrm{B}+$ \\
Des. & $0 \% \mathrm{~S}$ & $1 \% \mathrm{~S}$ & $2 \% \mathrm{~S}$ & $3 \% \mathrm{~S}$ & $4 \% \mathrm{~S}$ & $5 \% \mathrm{~S}$ \\
\hline $\mathrm{CP}$ & 25500 & 2920.0 & 3700.0 & 3850.0 & 4110.0 & 4200.0 \\
$\mathrm{SS}$ & 173.5 & 198.6 & 251.7 & 261.9 & 278.9 & 285.7 \\
$\mathrm{SR}$ & 6.800 & 6.8 & 6.8 & 6.8 & 6.8 & 6.8 \\
$\mathrm{~T} \%$ & 25.0 & 29.20 & 3.7 & 3.85 & 4.11 & 4.2 \\
\hline
\end{tabular}

Table 7: Bitumen $80 / 100$ Viscosity at $75^{\circ} \mathrm{C}$

\begin{tabular}{lllllll}
\hline & $\mathrm{B}+$ & $\mathrm{B}+$ & $\mathrm{B}+$ & $\mathrm{B}+$ & $\mathrm{B}+$ & $\mathrm{B}+$ \\
Des. $0 \% \mathrm{~S}$ & $1 \% \mathrm{~S}$ & $2 \% \mathrm{~S}$ & $3 \% \mathrm{~S}$ & $4 \% \mathrm{~S}$ & $5 \% \mathrm{~S}$ \\
\hline $\mathrm{CP}$ & 28100.0 & 28000.0 & 33100.0 & 34300.0 & 47100.0 & 49300.0 \\
SS 1911.6 & 1904.8 & 2251.7 & 2333.3 & 3204.0 & 3353.7 \\
SR 6.8 & 6.8 & 6.8 & 6.8 & 6.8 & 6.8 \\
T \% 28.1 & 28.1 & 33.1 & 34.1 & 47.1 & 49.3 \\
\hline
\end{tabular}

The Figure 1 below indicated a reduction in viscosity as the additive content was increased at $150^{\circ} \mathrm{C}$.

From the study the addition of $\mathrm{S}$ at $135^{\circ} \mathrm{C}$ temperature indicated a reduction in the viscosity of the binder as illustrated in Fig. 2.

From the Table 5 below the study indicates that the addition of $\mathrm{S}$ at $120^{\circ} \mathrm{C}$ resulted in a decrease in viscosity of the binders as the additive content was increased Fig. 3.

The increase in the additive also increased the viscosity at $100^{\circ} \mathrm{C}$ as shown in Table 6 .

Results from this study indicated increase in viscosity at midrange temperature of $75^{\circ} \mathrm{C}$, as presented in Table 7;

The viscosity test at $100^{\circ} \mathrm{C}$ indicated an increase viscosity as the additive was increased in the binder as could be seen in Fig. 4 below;

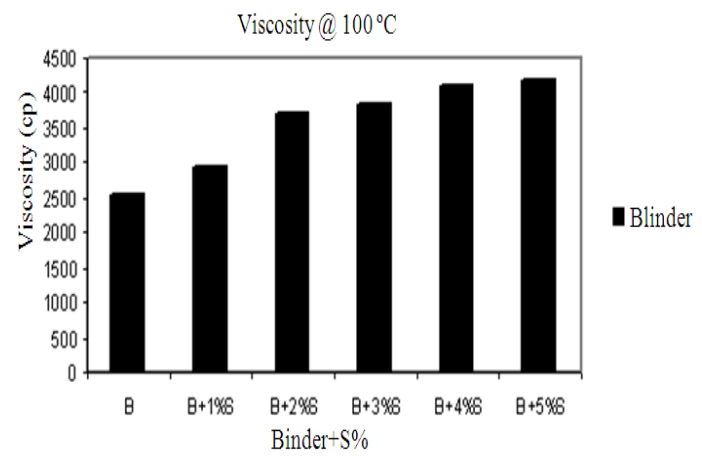

Fig. 4: Viscosity at $100^{\circ} \mathrm{C}$ 


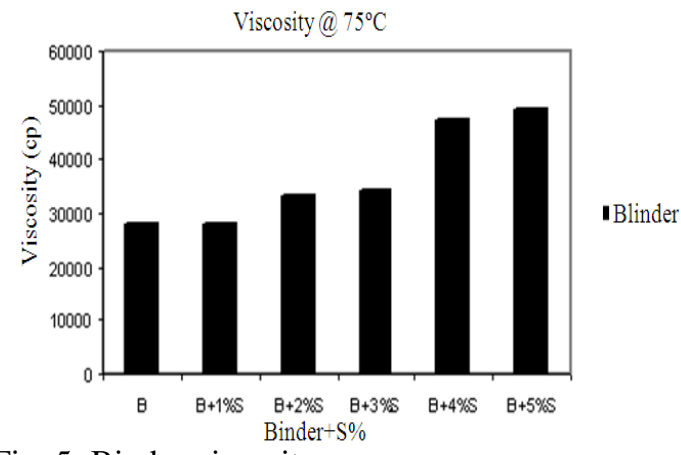

Fig. 5: Binder viscosity

From the study the addition of Sasobit to the binder at midrange temperature of $75^{\circ} \mathrm{C}$ when investigated indicated an increase in viscosity as shown in Fig 5 below.

Compatibility test: All the test samples of neat bitumen with the additive where tested for compatibility in accordance with the shell hand book code on compatibility test, the samples were put in the oven at $150{ }^{\circ} \mathrm{C}$ and the softening point of the top and bottom tested for 1day, 3days, 5days and 7days with all the samples producing a softening point difference of less than $5^{\circ} \mathrm{C}$ between the top and bottom for all the samples indicating that Sasobit is compatible and is storage stable with bitumen.

\section{CONCLUSION}

This study has indicated that the additive increases the softening point and reduces the penetration of the binder it also increases the viscosity of the binder at low temperature and decreases the viscosity at high temperature. The addition of Sasobit significantly reduces the viscosities of the binder at temperatures above $100^{\circ} \mathrm{C}$ there by easing production, reduce emission and providing low temperature at site (environments). This advantage has enabled the production of asphalt concrete at low temperatures, using the Warm Mix Asphalt technology (WMA), which is currently been investigated, at the highway laboratory at the university of technology Malaysia. The effect of Sasobit on the viscosity of the binder guarantees an efficient coating of the aggregate by the at lower temperatures compared to the conventional Hot Mix Asphalt (HMA). Also the addition of the additive at midrange temperature of $75^{\circ} \mathrm{C}$ indicated an increase in viscosity as the percentage of the additive was increased. It is a wax, which recrystallizes in the binder on cooling, there by increasing the viscosity of the binder at lower temperatures, this enhances the flow of the binder. Base on the penetration test conducted on the $2 \% \mathrm{~S}$ modified binder could be categorized as PG70-16.

\section{ACKNOWLEDGEMENT}

The study as described in this study was carried out in the department of highway and transportation, faculty of Civil engineering University Technology Malaysia (UTM). The commercial additive sasobit wax used in the study was supplied by Sasol international of South Africa, all are acknowledged.

\section{REFERENCES}

Gandhi, T. and S. Amirkhanian, 2008. Effect of warm asphalt additives on asphalt binder and mixture properties. Ph.D. Thesis, Clemson University, U.S.A. pp: 1-30.

Edwards, Y., Y. Tasdemir and U. Isacsson, 2006. Rheological effects of commercial waxes and phosporic acid in bitumen 160/220. High and Medium Temperature Performance. J. of Const. and Buildg. Mat., 21:1899-1908. DOI: 10.1016/j.conbuildmat.2006.07.012.

Edwards, Y., Y. Tasdemir and U. Isacsson, 2006. Effect of commercial waxes on asphalt concrete mixtures performance. J.Cold region Science and Technology 45: 32-41. DOI: 10.1016/j.coldregions.2006.01.002.

Kanitpong, K., Nam K., Martono W. and Bahia H., 2008. Evaluation of a warm mix asphalt additive. Proceedings of the Institution of Civil Engineers construction materials, 161: 1-8, DOI: 10.1680/coma.2008.161.1.1.

Kim, H., 2010. Performance Evaluation of SBS modified asphalt mixtures using Warm mix technologies. Ph.D. Thesis, Clemson University, U.S.A. pp: 1-25.

Graham, C.H. and Brian, D.P., 2005. Evaluation of sasobit for use in warm mix asphalt, NCAT Report 05-06,pp: 2-11.

Marandi, S. M.and Safapour, P., 2009. Base Course Modification through Stabilization using Cement and Bitumen. Am. J. Appli. Sci. 6: 30-42. DOI: 10.3844/ajassp.2009.30.42.

Maccorone, 1994. Warm mix asphalt for cold wheather paving. pp: 1-2 (retrieved 23/09/10 from www.swutc.tamu.edu/pub).

Xiaohu, L. and U. Issacsson, 2003. Rheological effect of waxes in bitumen. J. of Energy and Fuels, 17: 511-520. DOI: 10.1021/ef020202b.

Meor, O.,T. Ali and Z. shahadan, 2010.Evaluation of the potential of sasobit to reduce required heat energy and $\mathrm{C} 02$ emission in the asphalt industry. J. Cleaner Produc., 18: 1859-1865 DOI: $10.1016 /$ j.jclepro.2010.08.002 